\title{
Excitonic Polaritons in Semiconductor Micropillars
}

\author{
D. Bajoni, E. Wertz, P. Senellart, A. Miard, \\ E. Semenova, A. Lemaître, I. Sagnes, S. Bouchoule \\ AND J. BLOCH* \\ Laboratoire de Photonique et de Nanostructures, LPN/CNRS \\ Route de Nozay, 91460 Marcoussis, France
}

\begin{abstract}
We describe the physics of cavity polaritons in semiconductor micropillars. Cavity polaritons are exciton-photon entangled states arising from the strong coupling between excitons and the optical modes of a cavity. In micropillars, the photon three-dimensional confinement results in a discrete spectrum of $0 \mathrm{D}$ polariton states. Characterization of the linear properties of these micropillars will be presented. Then we will show how this system can be used to generate parametric photons and to obtain polariton lasing.
\end{abstract}

PACS numbers: 71.36+c, 78.45+h, 78.55.Cr, 78.67.Pt

\section{Introduction}

We consider a Fabry-Perot cavity defined by two Bragg mirrors and containing one or several quantum wells (QW), whose emission is resonant to the optical cavity mode. If the cavity finesse is strong enough, the photons emitted by the quantum well excitons can oscillate long enough within the cavity, to be reabsorbed, reemitted again and so on. The emission of light becomes reversible and the light-matter interaction has to be treated in a non-perturbative way. In this so-called strong coupling regime, the eigenstates of the system are exciton-photon entangled states, named cavity polaritons. First evidenced through reflectivity measurements in the pioneer work from Weisbuch et al. [1], the characteristic dispersion relations of cavity polaritons have been clearly evidenced by photoluminescence [2].

Cavity polaritons in two-dimensional systems have attracted a lot of interest these last years because of their non-linear properties [3]. Since cavity polaritons originate from the hybridization of two bosons, they obey Bose statistics in the low density regime. Stimulated scattering into a given polariton state can be

*corresponding author; e-mail: jacqueline.bloch@lpn.cnrs.fr 
triggered when its occupancy exceeds unity. This is the so-called "boser" effect or polariton laser [4]. Moreover, because of their photonic part, their effective mass is three orders of magnitude smaller than those of the bare exciton. As a result, the Bose-Einstein condensation of cavity polaritons has been predicted [5-8], and may be achievable at room temperature in GaN based samples [5]. Recently massive occupation of the lowest energy polariton states has been observed in planar semiconductor microcavities: polariton lasing with the onset of coherent emission under non-resonant excitation has been reported by several groups [9-12].

Another very interesting aspect of the polariton physics is their $\chi^{3}$ nonlinearities due to the strong exciton-exciton interactions in semiconductor quantum wells [13]. Polariton-polariton interactions can be used to achieve polariton parametric scattering and parametric amplification [14-17]. This is a promising way to generate quantum correlated photon pairs [18-22].

In the present paper we want to describe a different cavity geometry, namely micron-sized pillar cavities. In such a system, the polariton energy spectrum is not a quasi-continuum as in the $2 \mathrm{D}$ case, but is split into well defined discrete states. After a brief description of the sample fabrication, the demonstration of the strong coupling regime in micropillars will be described. Then we will show how polariton parametric scattering between adjacent polaritons modes can be obtained. Finally, polariton lasing will be demonstrated. Changing the excitation condition, we observe competition between polariton modes. We therefore conclude on polariton lasing and rule out Bose-Einstein condensation driven by thermodynamical equilibrium which should always occur on the lowest energy state.

Finally, we will conclude by underlining the advantage of the pillar system as compared to higher dimensionality cavities.

\section{Demonstration of the strong coupling regime}

Microcavity samples are grown by molecular beam epitaxy. They consist in two Bragg mirrors surrounding a cavity. Quantum wells resonant to the cavity mode are inserted at the antinodes of the electromagnetic field. Experiments performed on two cavity samples will be presented here. The characteristics of the different samples are summarized in the Table.

TABLE

Sample characteristics

\begin{tabular}{c|c|c|c}
\hline \hline Sample & Bragg mirrors & Cavity & QWs \\
\hline 1 & $\mathrm{Al}_{0.1} \mathrm{Ga}_{0.9} \mathrm{As} / \mathrm{AlAs}$ & $2 \lambda$ GaAs cavity & $3 \mathrm{In}_{0.05} \mathrm{Ga}_{0.95} \mathrm{As} \mathrm{QWs}$ \\
& Top/Bottom 20/24 pairs & & $(8 \mathrm{~nm})$ \\
2 & $\mathrm{Ga}_{0.05} \mathrm{Al}_{0.95} \mathrm{As} / \mathrm{Ga}_{0.80} \mathrm{Al}_{0.20} \mathrm{As}$ & $\lambda / 2 \mathrm{Ga}_{0.05} \mathrm{Al}_{0.95} \mathrm{As}$ & $12 \mathrm{GaAs} \mathrm{QWs}$ \\
& Top/Bottom 26/30 pairs & & $(7 \mathrm{~nm})$
\end{tabular}


For each sample, the cavity is wedge shaped so that the detuning $\delta=E_{\mathrm{c}}-E_{\mathrm{x}}$ between the cavity mode (energy $E_{\mathrm{c}}$ ) and the exciton transition (energy $E_{\mathrm{x}}$ ) can be varied by changing the spot under investigation on the wafer. Micropillars of size ranging from 20 to $2 \mu \mathrm{m}$ are designed by electron beam lithography and etched by reactive ion etching. A scanning electron micrography showing an array of such micropillars is presented in Fig. 1a.
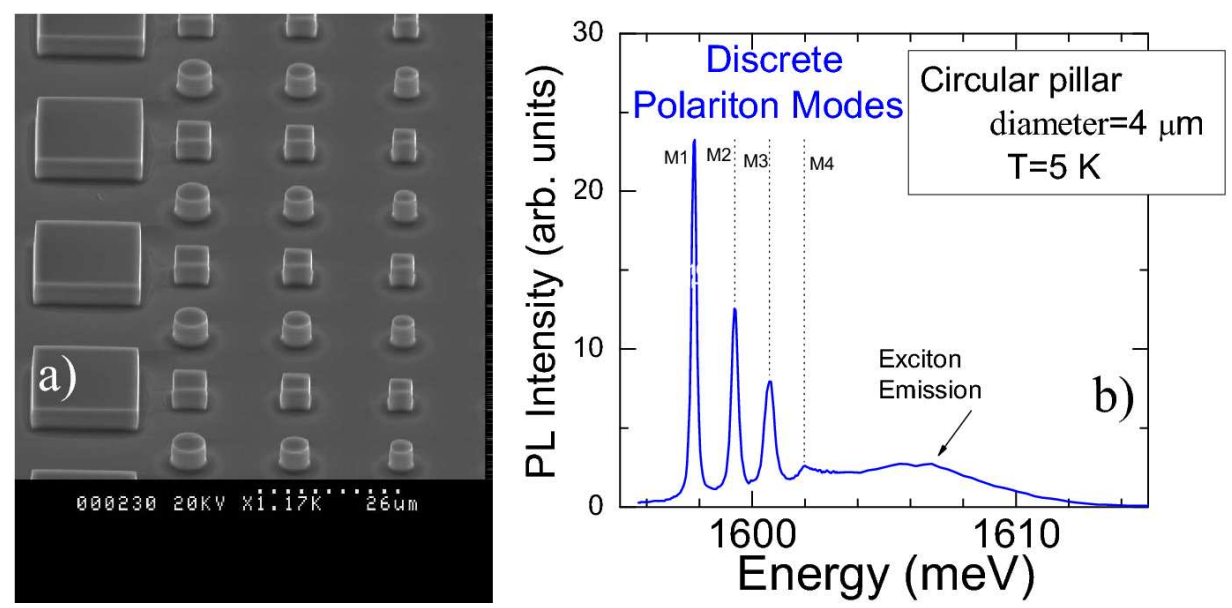

Fig. 1. (a) Scanning electron micrography showing a micropillar array; (b) microphotoluminescence spectrum of a $4 \mu \mathrm{m}$ diameter micropillar; discrete polariton modes as well the exciton line are clearly resolved.

Samples are maintained at low temperature in a cold finger cryostat. Microphotoluminescence on single micropillars is performed using a cw Ti:sapphire laser focused onto a $3 \mu \mathrm{m}$ diameter spot through a microscope objective. The micropillar emission is collected through the same objective, spectrally dispersed and analyzed with a nitrogen cooled CCD camera.

Figure 2b presents a PL spectrum measured on a single $4 \mu \mathrm{m}$ diameter circular micropillar on sample 2 . The emission energies measured on micropillars of identical diameter along the cavity wedge are summarized in Fig. 2. The emission spectrum in Fig. 1b presents several discrete emission lines on the low energy side of the exciton line centered around $1607 \mathrm{meV}$. The energy of these discrete lines strongly varies with the layer thickness: they are attributed to $0 \mathrm{D}$ photon modes $[23,24]$. Each of these photon modes presents an anticrossing [25, 26] with the exciton line characteristic of the strong coupling regime.

In pillar cavities, photons are confined vertically by the Bragg mirrors and laterally by the index of refraction contrast between air and semiconductor. For lateral dimensions superior to $1 \mu \mathrm{m}$, an easy way to calculate optical modes of such a micropillar is to treat the lateral confinement as a perturbation of the planar case. This lateral confinement breaks the in-plane translation invariance of the 


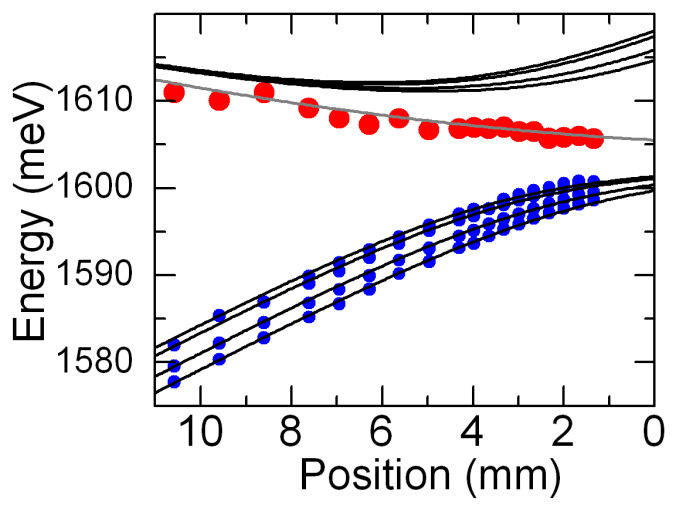

Fig. 2. Symbols - emission energy measured on different micropillars along the cavity wedge; black solid lines - calculated energy of the 0D polariton modes; gray line energy of the exciton.

cavity. In the case of rectangular micropillars, this results in a quantification of $k_{x}$ and $k_{y}$, the in-plane components of the photon wave vector [26]:

$$
k_{x}=\frac{2 \pi n_{x}}{L_{x}} \quad \text { and } \quad k_{y}=\frac{2 \pi n_{y}}{L_{y}},
$$

where $n_{x}$ and $n_{y}$ are integers, $L_{x}$ and $L_{y}$ are the lateral dimensions of the micropillars. To properly describe the radiative coupling between these 0D photon states and the QW exciton, exciton states have to be described in a basis with the proper symmetry [27]. Thus similarly to the $2 \mathrm{D}$ case where the proper basis is the basis of propagative planar waves, in micropillars stationary waves have to be considered. For rectangular pillars, the in-plane exciton envelope wave functions are described as

$$
\psi_{n_{x}, n_{y}}(x, y)=A \cos \left(\frac{\pi n_{x} x}{L_{x}}\right) \cos \left(\frac{\pi n_{y} y}{L_{y}}\right) .
$$

The case of circular pillars, where the Bessel functions have to be considered to include the cylindrical symmetry, has been treated in [23].

In the strong coupling regime, polaritons come from the mixing between each of these $0 \mathrm{D}$ photon modes and the QW exciton state of the same $n_{x}$ and $n_{y}$ [27].

The 0D polariton energies of Fig. 2 are well reproduced using a $15 \mathrm{meV}$ Rabi splitting, the value obtained in the planar cavity of this sample. Let us note that contrary to $2 \mathrm{D}$ cavities, the uncoupled exciton line is observed because in-plane exciton emission is extracted through the pillar sides. Since the exciton line is broadened by strain relaxation in the etching process, the upper polariton state could not be resolved.

Thus the variation of the emission line energies from one pillar to the other demonstrates the exciton-photon strong coupling regime and the formation of $0 \mathrm{D}$ polariton states. 


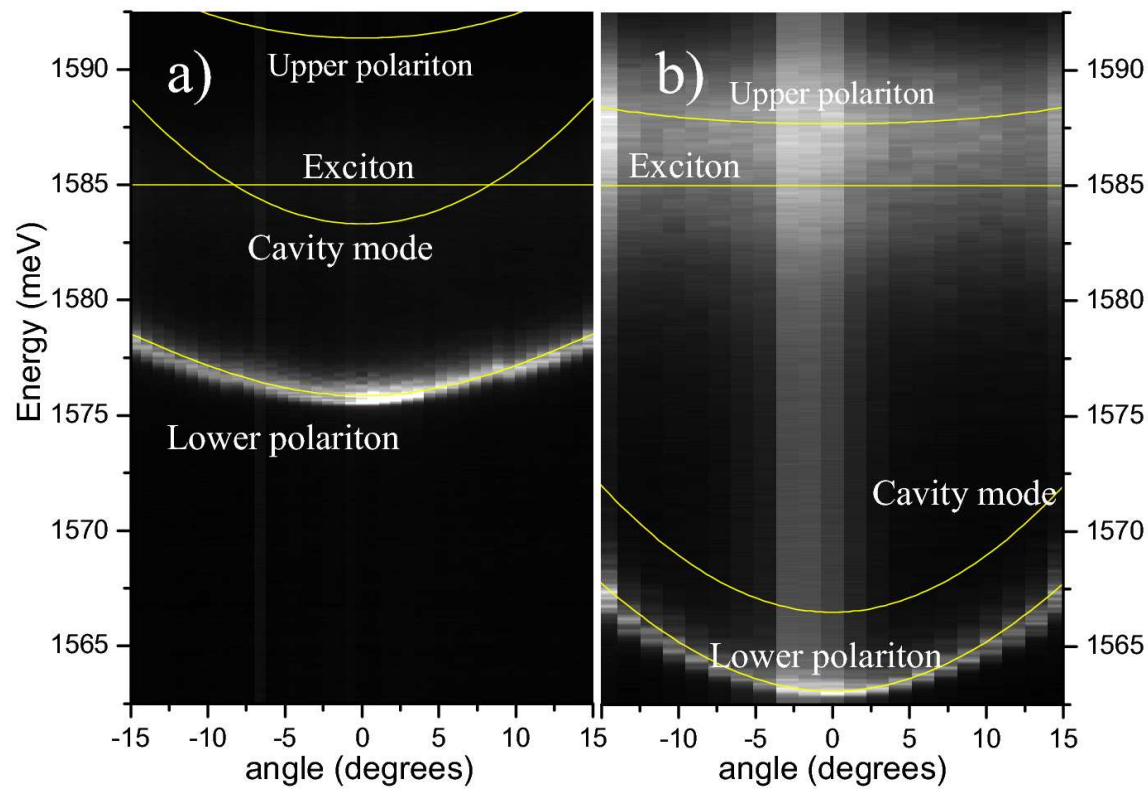

Fig. 3. Emission intensity as a function of the energy and of the angle measured on a $20 \mu \mathrm{m}$ square micropillar. Part (a) corresponds to a detuning $\delta=-2.7 \mathrm{meV}$ and (b) to $\delta=-21 \mathrm{meV}$. Solid lines - energy of the uncoupled exciton and cavity mode and of the upper and lower polariton branches.

Further confirmation is obtained by measuring the emission pattern of a single micropillar [28]. The emission angle $\theta_{n_{x}, n_{y}}$ of a mode of index $n_{x}, n_{y}$ is directly related to $k_{x}$ and $k_{y}$ using

$$
\sin \left(\theta_{n_{x}, n_{y}}\right)=\frac{\hbar c \sqrt{k_{x}^{2}+k_{y}^{2}}}{E_{n_{x}, n_{y}}} .
$$

Figures $3 \mathrm{a}$ and $3 \mathrm{~b}$ present the emission energy as a function of the detection angle on two $20 \mu \mathrm{m}$ diameter micropillars corresponding to two different detunings between the exciton and the photon modes. These measurements were performed with a $20 \mu \mathrm{m}$ diameter excitation spot and an angular resolution of $0.6^{\circ}$. Figure $3 \mathrm{~b}$ corresponds to a strong negative detuning $(\delta=-21 \mathrm{meV})$ : the observed polariton dispersion is that of the bare $2 \mathrm{D}$ cavity. The spectral separation of adjacent mode is sufficient so that the discrete modes can be distinguished. Figure 3a corresponds to a much smaller value of the detuning and the angular dispersion of the modes is strongly modified as compared to the bare cavity mode. This is the signature of the strong coupling regime with the exciton: the dispersion is well reproduced using a detuning $\delta=-2.7 \mathrm{meV}$. Thus these measurements highlight the formation of polariton states in micropillar cavities. This result was first demonstrated in 1997 by our group [25] and the group of Gutbrod et al. 
[26]. Recently other configurations have been proposed to laterally confine cavity polaritons. In Ref. [28], the cavity layer is etched into mesas before overgrowth of the Bragg mirror. This produces a shallower confinement than for micropillars but the polariton energy spectrum is clearly discretized. Another approach is to create a trap in real space by applying a local stress, with a tip pushing on the back side of a cavity sample [29]. In this case, polariton trapping has been observed in this local energy minimum, but the energy spectrum remains a quasi-continuum.

\section{Emission of parametric photons}

Because of their strong excitonic part, polariton Coulombian interaction results in strong optical nonlinearities [13]. In particular, polaritons resonantly pumped at the point of inflection of the dispersion relation efficiently scatter toward the energy minimum at $k=0$ (signal) and toward a higher energy state near the excitonic reservoir (idler) $[14,15]$. This parametric process conserves both energy and momentum. Considerable interest has been devoted in recent years to such polariton parametric scattering. Promisingly, nonclassical correlations are expected between the signal and idler beams [18-22]. In planar microcavities the idler state has a strongly excitonic character: idler polaritons are therefore less coupled to external radiation than signal polaritons, and they can scatter toward the excitonic reservoir of large $k$ states. The idler beam is therefore several orders of magnitude less intense than the signal beam, making signal and idler quantum correlations difficult to evidence [21].

As opposed to planar cavities, in micropillars the spectral separation between the exciton, and the three lowest energy polariton modes $\left(M_{1}, M_{2}\right.$, and $\left.M_{3}\right)$ with energies $E_{1}, E_{2}$, and $E_{3}$ can be made large enough to get negligible scattering toward the excitonic reservoir and approximatively equal coupling with the external radiation; of course the modes must retain a sufficient excitonic character to assure nonlinear properties. As a result, signal and idler beams are expected to be of similar intensities in a micropillar. Dasbach et al. evidenced polariton parametric scattering in square micropillars, with two complementary beams of comparable intensity [30]. These experiments were performed on an ensemble of micropillars and inhomogeneous broadening of the emission occurred due to variations of the micropillar size. Furthermore, quantum correlations exist only between signal and idler photons coming from the same micropillar. Recently we have demonstrated parametric luminescence on a single micropillar operating in the strong coupling regime [31]. We used a $3 \mu \mathrm{m}$ diameter micropillar designed on sample 1. To obtain parametric scattering from $M_{2}$ to $M_{1}$ and $M_{3}$, spectral equidistance between the modes has to be fulfilled: $E_{3}-E_{2}=E_{2}-E_{1}$. Fine tuning between the exciton and the photon modes is obtained through temperature changes. For the considered micropillar spectral equidistance is obtained at $53 \mathrm{~K}$ as shown in the photoluminescence spectrum in the inset of Fig. 4. The pump is then tuned to the energy of the second polariton mode $M_{2}$. Figure 4 presents 




Fig. 4. Inset: microphotoluminescence spectrum measured under non-resonant excitation on a $3 \mu \mathrm{m}$ diameter micropillar. $T=53 \mathrm{~K}$ to obtain spectral equidistance between the three lower energy polariton mode. Main figure: emission spectra normalized to the excitation power measured under excitation resonant to $M_{2}$, the second polariton mode. A quadratic increase in $M_{1}$ and $M_{3}$ is evidenced.

emission spectra normalized to the incident power under such resonant excitation. The quadratic increase in the emission intensity of both adjacent modes $M_{1}$ and $M_{3}$ is the signature of polariton-polariton parametric scattering [30]. These results are particularly promising since the integrated intensity of both signal and idler are of similar intensities. In this sample containing only 3 quantum wells within a $2 \lambda$ cavity, the parametric gain was not large enough to reach a population of 1 in mode $M_{1}$ and trigger stimulated parametric scattering.

Very recent preliminary measurements performed on sample 2 (more quantum wells, smaller cavity volume, higher finesse) indicates that this threshold can be reached on a single micropillar and that parametric oscillation can be achieved between discrete $0 \mathrm{D}$ polariton modes.

\section{Polariton laser}

Boson statistics can lead to massive occupation of a single quantum state and trigger final state stimulation. This stimulation is responsible for the bright coherent emission of light in a laser. Another fascinating property of massive bosons in thermal equilibrium is their ability to accumulate in the lowest energy state under a given critical temperature. First predicted in 1925 [32], the Bose-Einstein condensation was achieved in 1995 for ultracold atoms [33, 34]. Demonstrating such bosonic effects with matter waves in a solid-state system is interesting both from a fundamental point of view and for applications since it could provide a new source of coherent light. 
Massive occupation of a polariton state has been recently observed in semiconductor two-dimensional (2D) cavities and attributed to the Bose-Einstein condensation $[9,10]$ or to polariton lasing [11]. Polariton condensation has also been reported in a stress induced localized energy trap [12] with dimensions sufficiently large for the system to present a $2 \mathrm{D}$ continuum of states. In these experiments, the clear distinction of a thermodynamic phase transition (Bose-Einstein condensation) from a kinetic stimulated scattering (polariton lasing) is still debated.

We have recently observed stimulated scattering into a discrete polariton state in micropillars designed on sample 2 [35]. As an illustration of these measurements, we report here on experiments performed on a $3.2 \mu \mathrm{m}$ square micropillar. Figure 5a presents microphotoluminescence spectra measured on the micropillar under non-resonant excitation for several excitation powers. The integrated intensity is summarized in Fig. 5b. A marked threshold around $100 \mu \mathrm{W}$ is observed, above which the emission intensity of the lowest energy line abruptly increases by more than two orders of magnitude. Looking at the absolute emission intensity, we checked that at threshold the occupancy of the considered polariton state reaches unity [36]. The negligible energy shift at threshold indicates that the observed stimulated emission occurs in the strong coupling regime [37]. Onset of coherent emission is indicated by the spectral narrowing above threshold. Further increasing excitation power, the emission spectrum becomes broad and strongly blueshifts. This is the signature of the bleaching of the strong coupling and the progressive transition into the weak coupling regime [37]. A second threshold is then observed after the blueshift has saturated, associated to the onset of regular photon lasing in weak coupling. Observing the transition from strong to weak coupling clearly demonstrates that the observed stimulated emission at low power occurs on a polariton state.

We now want to address whether the observed feature is related to the Bose condensation (driven by thermodynamics) or to polariton lasing (driven by kinetics in an out of equilibrium system). The following experiment brings some insight into this issue. Figure 6a presents emission spectra from a $6 \mu \mathrm{m}$ micropillar excited through the edge. Contrary to the case of a well centered excitation where the stimulated emission occurs on the lowest energy polariton state, here the stimulated emission is first triggered on the second polariton mode. Then stimulated emission also occurs on the third and first polariton mode. Thus mode competition between polariton modes is observed. Depending on the precise excitation condition, a mode overcomes the others. This behavior is analogous to mode competition observed in any photon laser. It rules out the Bose-Einstein condensation which would always occur on the lowest energy states. We therefore conclude that our experiment is the first observation of polariton lasing on the zero-dimensional states of a micropillar. 

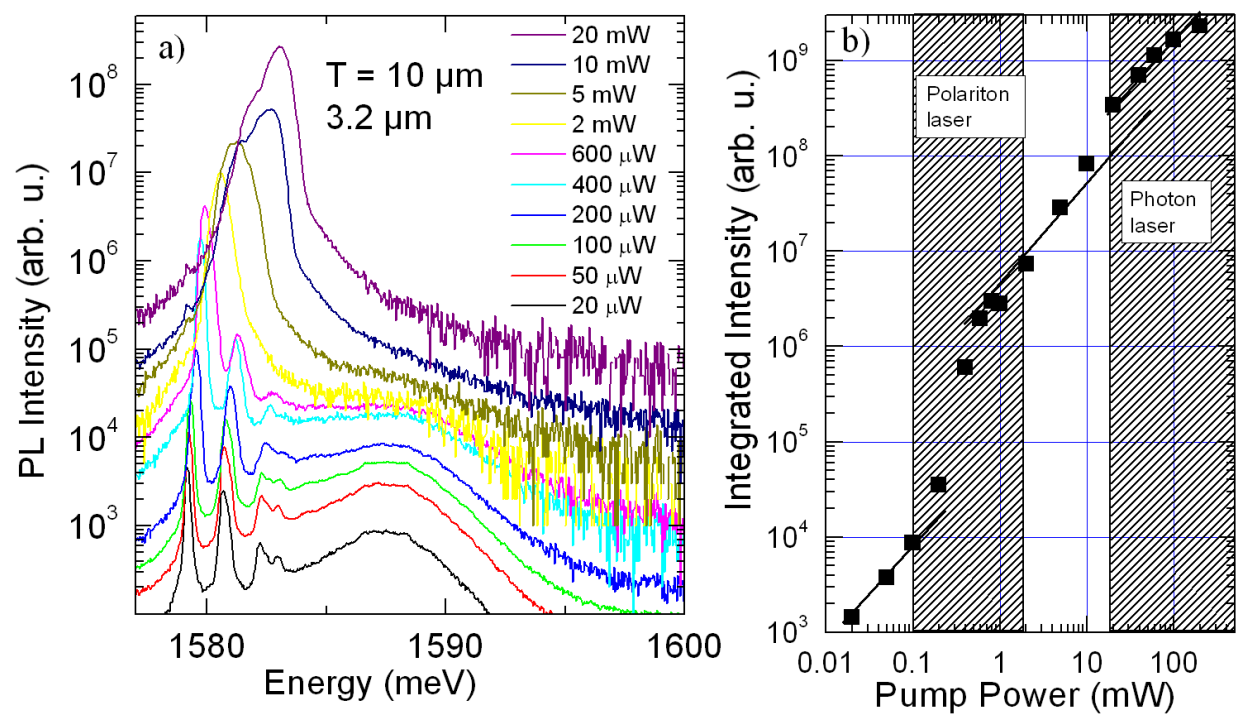

Fig. 5. (a) Microphotoluminescence spectra measured under non-resonant excitation on $3.2 \mu \mathrm{m}$ square micropillar for various excitation powers; (b) integrated emission intensity as a function of the excitation power, dashed areas indicate the excitation range for polariton laser and photon laser.
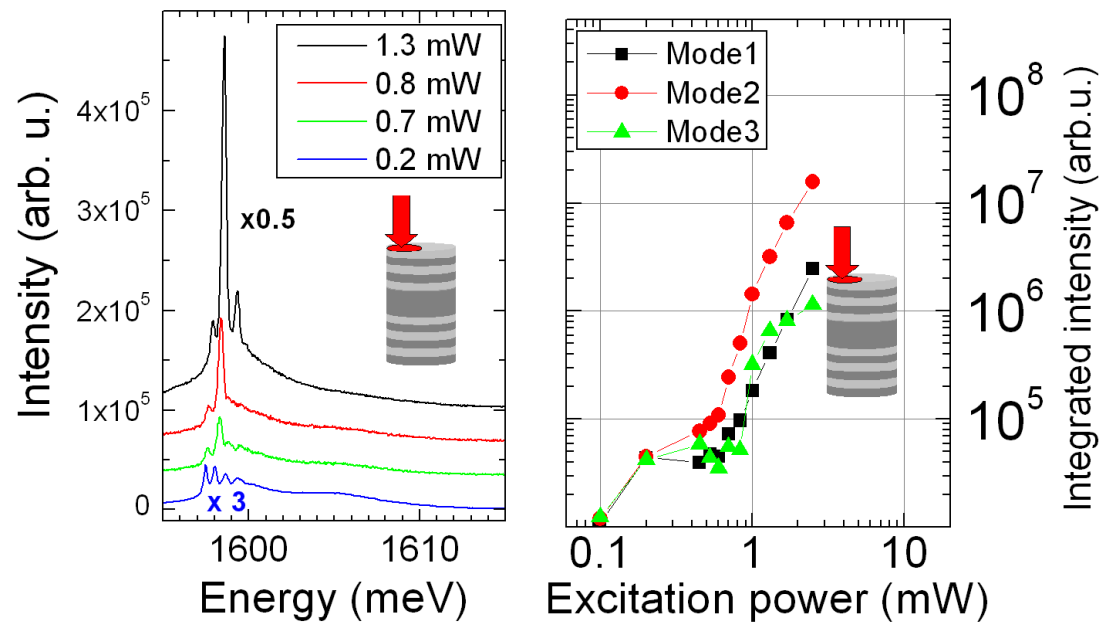

Fig. 6. (a) Microphotoluminescence spectra measured under non-resonant excitation at the edge of a $6 \mu \mathrm{m}$ micropillar; (b) integrated emission intensity of modes $M_{1}, M_{2}$ and $M_{3}$ under such edge excitation as a function of the excitation power. Mode competition is evidenced. 


\section{Conclusion}

We have shown that polariton states can be discretized by the photon three-dimensional confinement in semiconductor micropillars. Polariton parametric scattering as well as polariton lasing is observed on these 0D polariton states. Micropillars offer a unique system to study a well defined macroscopically occupied discrete state with a half matter half light wave. Moreover, since the density of state is considerably reduced as compared to a two-dimensional system, decoherence induced by scattering into the neighboring states is expected to be reduced. Further study is required to confirm that coherence and quantum correlations are more robust in micropillars.

\section{References}

[1] C. Weisbuch, M. Nishioka, A. Ishikawa, Y. Arakawa, Phys. Rev. Lett. 69, 3314 (1992).

[2] R. Houdré, C. Weisbuch, R.P. Stanley, U. Oesterle, P. Pellandini, M. Ilegems, Phys. Rev. Lett. 73, 2043 (1994).

[3] For a review see A. Kavokin, G. Malpuech, Cavity Polaritons, Elsevier, Amsterdam 2003.

[4] A. Imamoglu, R.J. Ram, S. Pau, Y. Yamamoto, Phys. Rev. A 53, 4250 (1996).

[5] G. Malpuech, A. Di Carlo, A. Kavokin, J.J. Baumberg, M. Zamfirescu, P. Lugli, Appl. Phys. Lett. 81, 412 (2002).

[6] Fabrice P. Laussy, G. Malpuech, A. Kavokin, P. Bigenwald, Phys. Rev. Lett. 93, 016402 (2004).

[7] J. Keeling, P.R. Eastham, M.H. Szymanska, P.B. Littlewood, Phys. Rev. Lett. 93, 226403 (2004).

[8] M.H. Szymanska, J. Keeling, P.B. Littlewood, Phys. Rev. Lett. 96, 230602 (2006).

[9] H. Deng, G. Weihs, C. Santori, J. Bloch, Y. Yamamoto, Science 298, 199 (2002).

[10] J. Kasprzak, M. Richard, S. Kundermann, A. Baas, P. Jeambrun, J.M.J. Keeling, F.M. Marchetti, M.H. Szymanska, R. André, J.L. Staehli, V. Savona, P.B. Littlewood, B. Deveaud, Le Si Dang, Nature (London) 443, 409 (2006).

[11] S. Christopoulos, G. Baldassarri Höger von Högersthal, A.J.D. Grundy, P.G. Lagoudakis, A.V. Kavokin, J.J. Baumberg, G. Christmann, R. Butté, E. Feltin, J.-F. Carlin, N. Grandjean, Phys. Rev. Lett. 98, 126405 (2007).

[12] R. Balili, V. Hartwell, D. Snoke, L. Pfeiffer, K. Westet, Science 316, 1007 (2007).

[13] C. Ciuti, P. Schwendimann, A. Quattropani, Phys. Rev. B 63, 041303 (2001).

[14] P.G. Savvidis, J.J. Baumberg, R.M. Stevenson, M.S. Skolnick, D.M. Whittaker, J.S. Roberts, Phys. Rev. Lett. 84, 1547 (2000).

[15] R.M. Stevenson, V.N. Astratov, M.S. Skolnick, D.M. Whittaker, M. Emam-Ismail, A.I. Tartakovskii, P.G. Savvidis, J.J. Baumberg, J.S. Roberts, Phys. Rev. Lett. 85, 3680 (2000).

[16] M. Saba, C. Ciuti, J. Bloch, V. Thierry-Mieg, R. André, Le Si Dang, S. Kundermann, A. Mura, G. Bongiovanni, J.L. Staehli, B. Deveaud, Nature 414, 731 (2001). 
[17] C. Diederichs, J. Tignon, G. Dasbach, C. Ciuti, A. Lemaître, J. Bloch, Ph. Roussignol, C. Delalande, Nature 440, 904 (2006).

[18] P. Schwendimann, C. Ciuti, A. Quattropani, Phys. Rev. B 68, 165324 (2003).

[19] C. Ciuti, Phys. Rev. B 69, 245304 (2004).

[20] S. Savasta, O. Di Stefano, V. Savona, W. Langbein, Phys. Rev. Lett. 94, 246401 (2005).

[21] J.Ph. Karr, A. Baas, E. Giacobino, Phys. Rev. A 69, 063807 (2004).

[22] M. Romanelli, C. Leyder, J.P. Karr, E. Giacobino, A. Bramati, Phys. Rev. Lett. 98, 106401 (2007).

[23] J.M. Gérard, D. Barrier, J.Y. Marzin, R. Kuszelewicz, L. Manin, E. Costard, V. Thierry-Mieg, T. Rivera, Appl. Phys. Lett. 69, 449 (1996).

[24] J.P. Reithmaier, M. Röhner, H. Zull, F. Schäfer, A. Forchel, P.A. Knipp, T.L. Reinecke, Phys. Rev. Lett. 78, 378 (1997).

[25] J. Bloch, R. Planel, V. Thierry-Mieg, J.M. Gerard, D. Barrier, J.Y. Marzin, E. Costard, Superlattices Microstruct. 22, 371 (1997).

[26] T. Gutbrod, M. Bayer, A. Forchel, J.P. Reithmaier, T.L. Reinecke, S. Rudin, P.A. Knipp, Phys. Rev. B 57, 9950 (1998).

[27] G. Panzarini, L.C. Andreani, Phys. Rev. B 60, 16799 (1999).

[28] R. Idrissi Kaitouni, O. El Daïf, A. Baas, M. Richard, T. Paraiso, P. Lugan, T. Guillet, F. Morier-Genoud, J.D. Ganière, J.L. Staehli, V. Savona, B. Deveaud, Phys. Rev. B 74, 155311 (2006).

[29] R.B. Balili, D.W. Snoke, L. Pfeiffer, K. West, Appl. Phys. Lett. 88, 031110 (2006).

[30] G. Dasbach, M. Schwab, M. Bayer, A. Forchel, Phys. Rev. B 64, 201309 (2001).

[31] D. Bajoni, E. Peter, P. Senellart, J.L. Smirr, I. Sagnes, A. Lemaître, J. Bloch, Appl. Phys. Lett. 90, 051107 (2007).

[32] A. Einstein, Sitzungsber. Preuss. Akad. Wiss. 1, 3 (1925).

[33] M.N. Anderson, J.R. Ensher, M.R. Matthews, C.E. Wieman, E.A. Cornell, Science 269, 198 (1995).

[34] K.B. Davis, M.-O. Mewes, M.R. Andrews, N.J. van Druten, D.S. Durfee, D.M. Kurn, W. Ketterle, Phys. Rev. Lett. 75, 3969 (1995).

[35] D. Bajoni, P. Senellart, E. Wertz, I. Sagnes, A. Miard, A. Lemaître, J. Bloch, Phys. Rev. Lett. 100, 047401 (2008).

[36] P. Senellart, J. Bloch, B. Sermage, J.Y. Marzin, Phys. Rev. B 62, R16263 (2000).

[37] R. Butté, G. Delalleau, A.I. Tartakovskii, M.S. Skolnick, V.N. Astratov, J.J. Baumberg, G. Malpuech, A. Di Carlo, A.V. Kavokin, J.S. Roberts, Phys. Rev. B 65, 205310 (2002). 